V. van Kampen

R. Merget

T. Brüning

\section{Berufliche Allergien gegen Papain}

\section{Occupational Allergies to Papain}

\section{Zusammenfassung}

Bereits seit längerem ist bekannt, dass die berufliche Exposition gegenüber Enzymstäuben pflanzlicher, bakterieller, mykotischer und tierischer Herkunft mit einem hohen Risiko einer spezifischen Sensibilisierung verbunden ist. Wie die hier vorgestellte Auswertung von Literaturdaten zeigt, trifft dies auch auf die industriell häufig eingesetzte aus der Papaya (Carica papaya) stammende Protease Papain zu. Durch eine größere Zahl von Studien sind Fälle von spezifischer Überempfindlichkeit der Atemwege oder der Lunge durch Papain gut belegt. Da Symptome, Hauttestergebnisse, der Nachweis spezifischer IgE-Antikörper und die Ergebnisse spezifischer Provokationstests in der Regel gut übereinstimmen, scheint ein immunologischer Wirkmechanismus gesichert.

\section{Abstract}

Occupational exposure against dusts of plant, bacterial, mould, and animal enzymes is long known to be associated with a high risk of specific sensitization. The present evaluation of literature data confirms that this is also true for papain. This frequently used industrial protease is derived from papaya (Carica papaya). Several cases of specific airway sensitization caused by papain are verified by a number of case reports and cross sectional studies. As symptoms, results of skin prick tests, detection of specific IgE-antibodies and results of specific bronchoprovocation tests are consistent, an immunologic mechanism can be assumed.

\section{Einleitung}

Papain ist eine Endopeptidase (EC 3.4.22.2) mit einem Molekulargewicht von ca. $23 \mathrm{kDa}$, die zusammen mit dem säurestabileren Chymopapain (EC 3.4.22.6) aus dem getrockneten Milchsaft (Latex) unreifer Papayas (Carica papaya) gewonnen wird. Ähnliche pflanzliche Enzyme sind das Bromelain aus Ananas und das Ficin aus Feigen. Da im aktiven Zentrum von Papain neben dem
Histidin auch ein Cysteinrest lokalisiert ist, gehört es zur Klasse der Thiolproteasen. Papain hat eine breite Substratspezifität; die Aminosäuren Arginin oder Lysin werden jedoch bevorzugt gebunden. Ähnlich wie bei den Serinproteasen erfolgt eine Inhibition durch anorganische Fluoride, Leupeptin oder Soyabohneninhibitor. Reagenzien mit freien Sulfhydrylgruppen wie Cystein, Glutathion oder Dithiothreitol aktivieren Papain, welches sogar noch in $0,1 \%$ iger Natriumdodecylsulfat-Lösung aktiv ist. Auf-

Serienherausgeber: $R$. Merget, D. Nowak

Institutsangaben

Berufsgenossenschaftliches Forschungsinstitut für Arbeitsmedizin (BGFA) Institut der Ruhr-Universität Bochum (Direktor: T. Brüning)

Anmerkung

Diese Arbeit ist eine modifizierte Version einer Publikation der Senatskommission der Deutschen Forschungsgemeinschaft (DFG) zur Prüfung gesundheitsschädlicher Arbeitsstoffe (erschienen in der 39. Ergänzungslieferung der Toxikologisch-arbeitsmedizinischen Begründungen von MAK-Werten, Wiley VCH, 2004).

Korrespondenzadresse

Dr. rer. nat. Vera van Kampen · BGFA · Bürkle-de-la-Camp-Platz 1 · 44789 Bochum · E-mail: kampen@bgfa.de 
grund seiner hohen Temperatur- und pH-Stabilität wird auf dieses Enzym bei vielen biotechnologischen Prozessen zurückgegriffen. So dient es als Fleischzartmacher in der Lebensmittelindustrie, zur Klärung von Getränken, als Eiweißfleckenentferner in der Waschmittelindustrie sowie in der Lederindustrie und bei der Herstellung von Zahnprothesereinigern. Darüber hinaus findet Papain in verschiedenen medizinischen Bereichen Anwendung. So werden ihm desinfizierende, blutstillende und heilungsfördernde Wirkungen zugesprochen.

Tiere mehrerer Arten entwickeln nach intratrachealer Instillation von Papain ein Lungenemphysem, das als Tiermodell für das menschliche Emphysem angewandt wird [1]. Beim Menschen hingegen sind im Zusammenhang mit der Exposition gegenüber Papain bislang nur Berichte über allergische Reaktionen, sowohl Kasuistiken als auch Querschnittuntersuchungen, veröffentlicht worden.

\section{Literaturauswertung}

Mithilfe geeigneter Stichwörter in sinnvoller Kombination wurden Veröffentlichungen in der Datenbank „Pubmed“ recherchiert. Uns im Original zugängliche relevante Publikationen über Studien zur sensibilisierenden Wirkung von Papain wurden hinsichtlich der klinischen Daten ausgewertet. Erfasst wurden neben der Zahl der exponierten Personen die Prävalenzen der einzelnen Symptome bzw. Diagnosen. Darüber hinaus wurde festgehalten, mit welchen Methoden (Hauttest, Bestimmung spezifischer IgE-Antikörper, Provokationstest) und unter Verwendung welcher Testlösungen eine auf Papain zurückzuführende Sensibilisierung diagnostiziert wurde.

\section{Ergebnisse}

Die Auswertung der Literatur zeigt, dass Papain ebenso wie vielen anderen Enzymen, z.B. Subtilisinen [2], Cellulasen [3] und Xylanasen [4] ein hohes Sensibilisierungspotenzial zukommt (Übersicht bei [5]). Neben der atemwegssensibilisierenden Wirkung wurden in einigen Fällen auch Hautsensibilisierungen durch papainhaltige Produkte beschrieben.

\section{Hautsensibilisierende Wirkung}

Zwei Kosmetikerinnen entwickelten nach dem Umgang mit papainhaltigen Reinigungstabletten auch eine Kontakturtikaria [6]. Im Rahmen eines anaphylaktischen Schocks durch Papain entwickelte ein 40-jähriger Mann an den Armen, Handgelenken, Oberschenkeln und im Gesicht Symptome von Urtikaria und Angioödem. Ein Scratchtest mit Papain verlief positiv [7]. Von neun Arbeitern, die beim Abfüllen und Verarbeiten direkten Kontakt mit papainhaltigen Fleischzartmachern hatten, klagten drei über Rötungen und Juckreiz an den exponierten Hautstellen [8].

\section{Atemwegssensibilisierende Wirkung}

Einen zusammenfassenden Überblick über die Ergebnisse der Literaturauswertung hinsichtlich der atemwegssensibilisierenden Wirkung von Papain gibt Tab. 1.

Tab 1 Klinische Studien zur atemwegssensibilisierenden Wirkung von Papain beim Menschen

\begin{tabular}{|c|c|c|c|c|c|}
\hline $\begin{array}{l}\text { Zahl der Un- } \\
\text { tersuchten }\end{array}$ & $\begin{array}{l}\text { arbeitsplatzbezogene Sympto- } \\
\text { me bzw. klinische Diagnosen }\end{array}$ & Pricktest (Testkonz.) & $\begin{array}{l}\text { Nachweismethode } \\
\text { spezif. IgE-Antikörper } \\
\text { (Methode) }\end{array}$ & $\begin{array}{l}\text { bronchiale Provokation } \\
\text { (Testkonz./Dosis) }\end{array}$ & Literatur \\
\hline 140 & $140 / 140 \mathrm{~A}, \mathrm{R}, \mathrm{K}$ & n.d. & $1 / 100$ positiv (RAST) & n.d. & 20 \\
\hline 124 & $70 / 124 \mathrm{~A}, \mathrm{R}, \mathrm{K}, \mathrm{H}$ & $\begin{array}{l}\text { 15/124 positiv (wässriger } \\
\text { Extrakt, k. w. A.) }\end{array}$ & $\begin{array}{l}\text { spez. Antikörper häufiger bei } \\
\text { Symptomatischen als bei Be- } \\
\text { schwerdefreien }\end{array}$ & n.d. & 21 \\
\hline 42 & 42/42 A,R,K,H,Haut & $\begin{array}{l}\text { 3/9 positiv } \\
\text { ( } 1-10 \mathrm{mg} / \mathrm{ml} \mathrm{Kochsalz,} \\
0,4 \% \text { Phenol, } 50 \% \text { Glyzerin) }\end{array}$ & 1/9 positiv (EAST) & $\begin{array}{l}1 / 9 \text { positiv } \\
\left(10^{-4}-5 \mathrm{mg} / \mathrm{ml} \text { Kochsalz/ }\right. \\
\text { Phenol-Lsg.) }\end{array}$ & 22 \\
\hline 33 & $17 / 33 \mathrm{~A} ; \mathrm{R}, \mathrm{K}, \mathrm{H}$ & $\begin{array}{l}\text { 16/33 positiv }(0,1 \mathrm{mg} / \mathrm{ml} \\
\text { Kochsalz/Phenol-Lsg.) }\end{array}$ & 15/33 positiv (RAST) & $\begin{array}{l}8 / 9 \text { positiv }(0,001-0,4 \mathrm{mg}) \\
5 \times \text { Sofortreak. } 3 \times \text { duale Reak. }\end{array}$ & 17 \\
\hline 31 & $17 / 31 \mathrm{~A}, \mathrm{R}$ & n.d. & $\begin{array}{l}\text { 13/31 positiv (darunter } 11 \\
\text { Symptomatische) }\end{array}$ & n.d. & 18 \\
\hline 23 & $\begin{array}{l}12 / 23 \mathrm{~A} \\
20 / 23 \mathrm{R} \\
17 / 23 \mathrm{~K} \\
20 / 23 \text { Haut }\end{array}$ & n.d. & 10/21 positiv (RAST) & n.d. & 16 \\
\hline 22 & k.w.A. & n.d. & $12 / 22$ positiv (CAP) & n.d. & 24 \\
\hline 22 & $\begin{array}{l}10 / 22 \mathrm{~A} \\
10 / 22 \mathrm{R} \\
3 / 22 \text { Haut }\end{array}$ & $\begin{array}{l}\text { 9/12 positiv }(0,01-10 \mathrm{mg} / \mathrm{ml} \\
\text { Kochsalz/Phenol-Lsg.) }\end{array}$ & $8 / 12$ positiv (RAST) & $\begin{array}{l}2 / 4 \text { positiv }(0,001-0,1 \mathrm{mg}) \\
2 \times \text { duale Reak. }\end{array}$ & 8 \\
\hline 11 & $7 / 11 \mathrm{~A}, \mathrm{R}$ & $\begin{array}{l}\text { 7/7 positiv }(0,1 \mathrm{mg} / \mathrm{ml} \text { Koch- } \\
\text { salz/Phenol-Lsg.) }\end{array}$ & 6/7 deutlich positiv (RAST) & $\begin{array}{l}5 / 5 \text { positiv }(0,1-1 \mathrm{mg} / \mathrm{ml}) \\
4 \times \text { Sofortreak. } 1 \times \text { duale Reak. } \\
(0,15-0,5 \mathrm{mg})\end{array}$ & 14 \\
\hline 10 & $\begin{array}{l}\text { 9/10 A,R,K,Haut beim } \\
\text { Umgang mit Weizenmehl }\end{array}$ & n.d. & 4/10 positiv (RAST) & n.d. & 27 \\
\hline
\end{tabular}




\begin{tabular}{|c|c|c|c|c|c|}
\hline $\begin{array}{l}\text { Zahl der Un- } \\
\text { tersuchten }\end{array}$ & $\begin{array}{l}\text { arbeitsplatzbezogene Sympto- } \\
\text { me bzw. klinische Diagnosen }\end{array}$ & Pricktest (Testkonz.) & $\begin{array}{l}\text { Nachweismethode } \\
\text { spezif. IgE-Antikörper } \\
\text { (Methode) }\end{array}$ & $\begin{array}{l}\text { bronchiale Provokation } \\
\text { (Testkonz./Dosis) }\end{array}$ & Literatur \\
\hline 10 & $\begin{array}{l}5 / 10 \mathrm{~A} \\
3 / 10 \mathrm{R} \\
1 / 10 \mathrm{~K} \\
3 / 10 \mathrm{H}\end{array}$ & $\begin{array}{l}9 / 10 \text { positiv }(0,01-1 \mathrm{mg} / \mathrm{ml} \\
0,9 \% \text { ige Kochsalzlösung) }\end{array}$ & 9/10 positiv (RAST) & n.d. & 26 \\
\hline 4 & $2 / 4 \mathrm{~A}, \mathrm{R}, \mathrm{K}, \mathrm{H}$ & $\begin{array}{l}\text { 2/2 positiv (Scratch, mit } \\
\text { Papainpaste (Papainpulver in } \\
\text { Coca-Lösung)) }\end{array}$ & n.d. & n.d. & 10 \\
\hline 2 & 2/2 A,R,K,Haut & $\begin{array}{l}2 / 2 \text { positiv ( } 5 \% \text { ige Papainlö- } \\
\text { sung in } P B S \text { ) }\end{array}$ & 2/2 positiv (CAP) & n.d. & 6 \\
\hline 2 & $2 / 2 \mathrm{~A}, \mathrm{R}, \mathrm{K}, \mathrm{H}$ & $\begin{array}{l}2 / 2 \text { positiv }(0,1-10 \mathrm{mg} / \mathrm{ml} \\
\text { PBS) }\end{array}$ & 2/2 positiv (RAST) & n.d. & 12 \\
\hline 1 & $\begin{array}{l}\text { H,Haut } \\
\text { anaphylakt. Schock }\end{array}$ & positiv (Scratch) & n.d. & n.d. & 7 \\
\hline 1 & $\mathrm{R}, \mathrm{K}$ & positiv $(0,01 \mathrm{mg} / \mathrm{ml})$ & n.d. & $\begin{array}{l}\text { positiv }{ }^{\mathrm{n}}(1 \mathrm{mg} / \mathrm{ml} \text { auf Baum- } \\
\text { wolle) }\end{array}$ & 23 \\
\hline 1 & $A, R, K$ & $\begin{array}{l}\text { positiv ( } 10 \mathrm{mg} / \mathrm{ml} \text { wässrige } \\
\text { Lsg., } 0,4 \% \text { Phenol, } 50 \% \\
\text { Glyzerin) }\end{array}$ & positiv (EAST, RAST-KI. 4) & n.d. & 25 \\
\hline 1 & $\mathrm{R}$ & positiv (2 plus) & n.d. & n.d. & 9 \\
\hline 1 & $\begin{array}{l}\text { A } \\
\text { (1 weitere Person verstarb an } \\
\text { anaphyl. Schock) }\end{array}$ & $\begin{array}{l}\text { positiv ( } 1 \mathrm{mg} / \mathrm{ml} \text { Kochsalz/ } \\
\text { Phenol-Lsg.) }\end{array}$ & n.d. & $\begin{array}{l}\text { Spirometrie am Arbeitsplatz } \\
\mathrm{FEV}_{1}: 73 \% \text { vom Sollwert }\end{array}$ & 11 \\
\hline 1 & $\mathrm{~A}, \mathrm{H}$ & $\begin{array}{l}\text { positiv (Scratch } 1 \mathrm{mg} / \mathrm{ml} \text {, In- } \\
\text { tradermal } 0,001-0,1 \mathrm{mg} / \mathrm{ml} \\
\text { Kochsalzlösung) }\end{array}$ & $\begin{array}{l}\text { positiv nach Entfernung von } \\
\lg G, \lg A, \lg M(R A S T)\end{array}$ & $\begin{array}{l}\text { positiv, duale Reaktion (Spin- } \\
\text { haler }^{\circledR}: 5 \text { mg Papain, } 195 \text { mg } \\
\text { Laktose) }\end{array}$ & 13 \\
\hline 1 & $A, R$ & $\begin{array}{l}\text { positiv ( } 1 \mu \mathrm{g} / \mathrm{ml} \text { PBS/Phenol- } \\
\text { Lsg.) }\end{array}$ & $\begin{array}{l}\text { positiv für Chymopapain } \\
\text { (ELISA) } \\
\text { Papain n.d. }\end{array}$ & n.d & 19 \\
\hline
\end{tabular}

Abkürzungen und Fußnoten:

$\mathrm{N} / \mathrm{n}$ absolute Zahl der pos. Reaktionen bezogen auf die Zahl der Untersuchten

Arbeitsplatz-bezogene Symptome: ARKH Atemwegsbeschwerden und/oder Rhinitis und/oder Konjunktivitis und/oder Husten

Bei der Provokation handelt es sich um bronchiale Provokationen, wenn nicht anders vermerkt ( ${ }^{\mathrm{n}}$ nasale Provokation)

k. w. A. keine weiteren Angaben, n.d. nicht durchgeführt

Eine berufsbedingte Atemwegsallergie gegen Papain wurde bereits 1931 bei einem Drogisten beschrieben. Der Mann klagte zunächst über Rhinitis, später über Asthma. Im Hauttest mit Papain zeigte der Patient eine positive Reaktion [9].

Zwei beruflich gegenüber Papain exponierte 27- bzw. 32-jährige Chemiker klagten über arbeitsplatzbezogene Atemwegsbeschwerden sowie über Rhinitis, Konjunktivitis und Husten. Im Scratchtest mit einer Papainpaste entwickelte sich in beiden Fällen innerhalb von 10 Minuten aus einem ca. 3-5 mm langen „Scratch“ eine deutliche Quaddel mit Pseudopodien und einem Durchmesser von ca. $9 \mathrm{~mm}$. 100 Kontrollpersonen zeigten im Scratchtest mit der Papainpaste keine Reaktion. Zur Herstellung der Paste wurde so lange Papainpulver zu $20 \mu$ l Coca-Lösung gegeben, bis eine pastöse Masse entstand. Zwei weitere Personen, die in einer Mühle beschäftigt waren, in der grobkörniges Papain zu feinem Pulver gemahlen wurde, berichteten lediglich von einmaligen Beschwerden $(1 \times$ Konjunktivitis bzw. $1 \times$ leichter Husten) beim Umgang mit Papain [10].

Ein 45-jähriger Mann klagte nach beruflichem Umgang mit Papainpulver beim Mischen und Verpacken von papainhaltigem Fleischzartmacher über Atemwegsbeschwerden. Im Pricktest mit Papainlösung in einer Konzentration von $1 \mathrm{mg} / \mathrm{ml} \mathrm{kam}$ es nach 20 Minuten zu einer deutlichen Reaktion mit einem Quaddeldurchmesser von $7 \mathrm{~mm}$. Keine Reaktion zeigte sich dagegen mit einer 10-fach höher konzentrierten Papainlösung bei mehreren atopischen und nicht-atopischen Kontrollpersonen. Bei der spirometrischen Untersuchung am Arbeitsplatz erreichte der Patient ein forciertes exspiratorisches Volumen in der ersten Sekunde $\left(\mathrm{FEV}_{1}\right)$, das lediglich $73 \%$ des errechneten Sollwertes ausmachte. Beschrieben wird zusätzlich der Fall eines 35-jährigen Mannes, der an den Folgen eines durch Papain verursachten anaphylaktischen Schocks am Arbeitsplatz verstarb [11].

Eine 24-jährige Laborantin einer Blutbank litt nach beruflicher Papain-Exposition unter Konjunktivitis und bereits zuvor unter Atemwegsbeschwerden beim Kontakt mit Katzen. Bei einem 27-jährigen Arbeiter, der mit dem Verladen von Papainpulver betraut war, traten Konjunktivitis, Rhinitis und Husten auf. Im Pricktest mit Papain reagierte die Laborantin auf die höchste Konzentration von $10 \mathrm{mg} / \mathrm{ml}$. Bei dem zweiten Patienten zeigten sich bereits mit den stärker verdünnten Papainlösungen $(0,1 \mathrm{mg}$ / $\mathrm{ml}$ bzw. $1,0 \mathrm{mg} / \mathrm{ml}$ ) deutliche Quaddeln. IgE-Antikörper gegen Papain konnten mittels Radio Allergo Sorbent Test (RAST) bei beiden Patienten nachgewiesen werden. Sowohl der Pricktest 
als auch der IgE-Nachweis waren bei 14 bzw. 18 weiteren Mitarbeitern der Blutbank negativ. Die routinemäßige Untersuchung von 330 Patienten einer allergologischen Klinik ergab in sieben Fällen $(2,1 \%)$ einen positiven Pricktest mit Papain. Keine dieser Personen berichtete jedoch über Beschwerden bei dem Umgang mit Papain. Bei vier von sechs hauttestpositiven Patienten konnten im Serum Papain-spezifische IgE-Antikörper detektiert werden [12].

Ein 58-jähriger Mann entwickelte nach 22-jähriger Tätigkeit in einer Fabrik zur Herstellung von papainhaltigem Fleischzartmacher, einer Zucker-Salz-Mischung mit 1\% Papain, Husten und Atemwegsbeschwerden. Der Hauttest verlief sowohl als Scratchtest mit $1 \mathrm{mg}$ Papain/ml als auch als Intrakutantest mit 0,001 - 0,1 mg Papain/ml positiv, bei zwei Kontrollpersonen dagegen negativ. Ein Prausnitz-Küstner-Test ergab ebenfalls ein positives Ergebnis. Der Nachweis Papain-spezifischer IgE-Antikörper mittels RAST lieferte zunächst für das Serum des Patienten einen vergleichbaren Wert $(290 \mathrm{cpm})$ wie für 15 Seren nicht-exponierter Kontrollpersonen $(320 \mathrm{cpm})$. Nach partieller Entfernung von IgG-, IgM- und IgA-Antikörpern aus den Seren ergab sich für das Patientenserum ein signifikant höherer Wert (6990 cpm) als für die Kontrollseren (570 cpm), was das Vorhandensein Papain-spezifischer IgE-Antikörper bestätigt. Papainspezifische IgG-Antikörper wurden mittels Ouchterlony-Test im Patientenserum nachgewiesen, in den Seren von 40 nicht-exponierten Kontrollpersonen jedoch nicht. Im bronchialen Provokationstest mittels Spinhaler ${ }^{\circledR}$ führte die Applikation von $5 \mathrm{mg}$ Papain vermischt mit $195 \mathrm{mg}$ Laktose zu einer dualen Reaktion. Ein Provokationstest mit Laktose bzw. dem Fleischzartmacher ohne Papain führte zu keiner bronchialen Reaktion [13].

Von neun Angestellten einer Industrieküche, in der Papain als Fleischzartmacher verwendet wurde und zwei Arbeitern der pharmazeutischen Industrie klagten sieben über arbeitsplatzbezogene bronchiale $(n=6)$ und/oder nasale $(n=5)$ Beschwerden. Alle sieben symptomatischen Patienten zeigten im Pricktest mit 0,1 mg Papain/ml eine ein- bis vierfach positive Reaktion. Vier asymptomatische exponierte Arbeiter sowie 40 nicht-exponierte asthmatische Kontrollpersonen reagierten im Pricktest mit einer 10-fach höher konzentrierten Papainlösung nicht. Papain-spezifische IgE-Antikörper waren in den Seren der sechs Arbeiter, die über berufsbedingte Atemwegsbeschwerden klagten, mittels RAST deutlich nachweisbar. Im Serum des symptomatischen Patienten, der lediglich an Rhinitis litt sowie in den Seren von zwei nicht-exponierten asthmatischen Kontrollpersonen ergab der RAST ein schwach positives Ergebnis. Im bronchialen Provokationstest mit 0,1-1,0 mg Papain/ml kam es bei allen fünf untersuchten Patienten bei einer Dosis zwischen 0,15 und 0,5 mg Papain zu einer Sofortreaktion mit einem Abfall der spezifischen Atemwegsleitfähigkeit $\left(\mathrm{sG}_{\mathrm{aw}}\right)$ von mindestens $50 \%$. In einem Fall kam es nach 3,5 Stunden zu einer zusätzlichen Spätreaktion [14]. Je zwei und drei der sechs Papain-sensibilisierten Personen reagierten im Pricktest auch auf Testzubereitungen mit $1 \mathrm{mg} / \mathrm{ml}$ bzw. $10 \mathrm{mg} / \mathrm{ml}$ der Ananasprotease Bromelain. Bei 5 der 6 Personen fanden sich auch positive Befunde im RAST. Zusätzlich verlief die bei zwei gegen Papain sensibilisierten Personen durchgeführte bronchiale Provokation mit $0,5 \mathrm{mg}$ Bromelain positiv. Die Autoren vermuten eine Kreuzreaktion zwischen den beiden Pflanzenproteasen [15].
In einer weiteren Studie klagten 12 von 23 symptomatischen Beschäftigten eines pharmazeutischen Unternehmens über Atemwegsbeschwerden, die übrigen über Rhinitis, Konjunktivitis oder Hautbeschwerden. Bei 17 Personen wurden Lungenfunktionsprüfungen, bei 21 Personen eine IgE-Bestimmung mittels RAST vorgenommen. Papain-spezifische IgE-Antikörper waren bei 10 der 21 Beschäftigten nachweisbar. In acht Fällen handelte es sich dabei um Personen mit Atemwegsbeschwerden. Die Untersuchungen ergaben eine signifikante Korrelation zwischen dem Vorhandensein bzw. der Menge Papain-spezifischer IgE-Antikörper und der Abnahme von $\mathrm{FEV}_{1}, \mathrm{FEF}_{75-85}$ (mittlerer forcierter exspiratorischer Fluss zwischen $75 \%$ und $85 \%$ der forcierten Vitalkapazität, FVC), RV (Residualvolumen) und TLC (totale Lungenkapazität) [16].

Von 22 in zwei Küchenbetrieben $(n=16)$ bzw. einer Gewürzmühle Beschäftigten klagten 12 Beschäftigte über Atemwegsbeschwerden, Rhinitis oder Exanthem. Im Pricktest mit 0,01 - $10 \mathrm{mg}$ Papain/ml reagierten neun der 12 symptomatischen Beschäftigten. Davon wiesen wiederum acht Beschäftigte im RAST Papain-spezifische IgE-Antikörper auf. In drei Fällen ließ sich ein ausgeprägtes Asthma bronchiale auf eine Papain-Sensibilisierung zurückführen. Diese wurde in zwei Fällen durch einen positiven bronchialen Provokationstest bestätigt. In einem Fall kam es nach Inhalation von 0,001 mg Papain zu einem Anstieg des Atemwegswiderstandes $\left(\mathrm{R}_{\mathrm{aw}}\right)$ von $0,24 \mathrm{kPa} / \mathrm{l} / \mathrm{s}$ auf $0,85 \mathrm{kPa} / \mathrm{l} / \mathrm{s}$. Im anderen Fall stieg der $\mathrm{R}_{\mathrm{aw}}$ nach Inhalation von $0,06 \mathrm{mg}$ Papain von $0,25 \mathrm{kPa} / \mathrm{l} / \mathrm{s}$ auf $1,61 \mathrm{kPa} / \mathrm{l} / \mathrm{s}$ an. Zwei weitere Personen reagierten im bronchialen Provokationstest auf 0,1 mg Papain lediglich grenzwertig $\left(\mathrm{R}_{\mathrm{aw}}\right.$-Anstieg um 0,07 bzw. $0,25 \mathrm{kPa} / \mathrm{l} / \mathrm{s})[8]$.

Von 33 beruflich exponierten Arbeitern (darunter vier hochexponierte Beschäftigte einer Gewürzmühle, 22 mäßig exponierte Beschäftigte aus zwei Industrieküchen oder aus der Herstellung von Papain-haltigen Digestiva sowie sieben nur indirekt exponierte Beschäftigte) klagten 17 (52\%) über Atemwegsbeschwerden, Rhinitis, Konjunktivitis oder Husten beim Umgang mit Papain. Der Pricktest mit 0,1 mg Papain/ml verlief in 16 Fällen positiv. Papain-spezifische IgE-Antikörper konnten mittels RAST bei 15 Arbeitern nachgewiesen werden. Bei 14 der asymptomatischen Beschäftigten waren sowohl der Pricktest als auch der Nachweis von spezifischem IgE negativ. Im bronchialen Provokationstest mit Papain reagierten acht von neun getesteten symptomatischen Patienten positiv. Dabei kam es fünfmal zu einer Sofortreaktion und dreimal zu einer dualen Reaktion [17]. In einer späteren Veröffentlichung zu den Befunden bei 31 dieser $33 \mathrm{Be}-$ schäftigten wurde angegeben, dass bei 13 Personen, darunter 11 Personen mit Beschwerden, Papain-spezifische IgE- und IgG-Antikörper nachzuweisen waren. Aus den Untersuchungen ging hervor, dass gegen Papain exponierte Arbeiter mit expositionsabhängigen Symptomen signifikant höhere Konzentrationen an Papain-spezifischen IgE- und IgG-Antikörpern aufwiesen als 14 nicht-exponierte Personen einer Kontrollgruppe $(p=0,001)$ und 14 asymptomatische Beschäftigte $(p=0,001)$ [18].

Eine 25-jährige Frau, die in der Gewinnung von Chymopapain aus Rohextrakt tätig war, klagte über arbeitsplatzbezogene Atemwegsbeschwerden und Rhinitis. Im Pricktest zeigte sie eine 
vierfach positive Reaktion auf $1 \mu \mathrm{g}$ Papain/ml. Bei 64 Kontrollpersonen verlief der Hauttest negativ [19].

In einer Studie wurden 140 Personen untersucht, die mindestens sechs Monate in einem Backbetrieb tätig waren und über berufsbedingte Beschwerden wie Asthma, Rhinitis oder Konjunktivitis klagten. Mittels RAST wurden die Patientenseren auf das Vorhandensein spezifischer IgE-Antikörper gegen verschiedene, in der Backindustrie verwendete Enzyme untersucht. In einem von 100 getesteten Seren waren spezifische IgE-Antikörper gegen Papain nachweisbar [20].

Ein 40-jähriger Mann kam mit Urtikaria und Angioödem als Anzeichen auf einen anaphylaktischen Schock in die Ambulanz. Neben der Verabreichung von Epinephrin wurde die 10-tägige Einnahme von Prednisolon verordnet. Nach Beendigung der Prednisoloneinnahme kam der Patient mit den zuvor genannten Symptomen sowie zusätzlich Husten, Glottis-Verengung und Hämaturie erneut in die Ambulanz. Die Symptome konnten mit der beruflichen Exposition gegenüber Papainpulver im Rahmen der Zahnpastaherstellung in Verbindung gebracht werden. Der Scratch-Test mit Papain verlief positiv [7].

Im Rahmen einer Querschnittstudie wurden 124 Beschäftigte der enzymverarbeitenden Abteilung eines Chemiebetriebes untersucht. 61 Personen waren in der Herstellung der Enzyme aus Aspergillus niger, A. oryzae und Bacillus subtilis, 42 Personen in der Weiterverarbeitung von Enzymen zu Wasch- und Backmitteln und 21 Personen in Laboratorien beschäftigt. Insgesamt klagten 70 Beschäftigte (56,5\%) über arbeitsplatzbezogene Beschwerden wie Rhinitis, Konjunktivitis, Husten oder Luftnot. Diese Beschwerden wurden bei Beschäftigten der Enzymherstellung und -weiterverarbeitung deutlich häufiger angegeben als von den Beschäftigten in den Laboratorien. Der Pricktest mit wässriger Papainlösung ergab bei 15 Beschäftigten ein positives Ergebnis. Spezifische IgE-Antikörper gegen Enzyme fanden sich häufiger bei Beschäftigten mit arbeitsplatzbezogenen und arbeitsplatzunabhängigen Atemwegsbeschwerden als in der beschwerdefreien Gruppe (keine weiteren Angaben) [21]. Bei 42 dieser Beschäftigten, die alle unter arbeitsplatzbezogenen Beschwerden litten, wurden in einer späteren Untersuchung insgesamt 82 bronchiale Provokationstests mit neun verschiedenen Enzymen durchgeführt. Eine von neun Provokationen mit Papain in aufsteigender Konzentration von $10^{-4}$ bis $5 \mathrm{mg} / \mathrm{ml}$ ergab ein positives Ergebnis mit einem mindestens 50\%igen Abfall der $s G_{a w}$ bei einer Papainaufnahme von $1 \mu \mathrm{g}$. Ein Pricktest mit Papainlösung ( $<1 \mathrm{mg} / \mathrm{ml}$ ) war ebenfalls positiv und mittels Enzym Allergo Sorbent Test (EAST) konnten nur im Serum dieses Beschäftigten Papain-spezifische IgE-Antikörper detektiert werden [22].

Eine 30-jährige Kosmetikerin litt nach dem Umgang mit einem papainhaltigen Pulver unter Rhinitis und Konjunktivitis. Der Pricktest mit 0,01 mg Papain/ml verlief positiv. Ebenfalls positiv verlief die nasale Provokation, bei der ein mit einer Papainlösung $(1 \mathrm{mg} / \mathrm{ml})$ getränkter Baumwollstreifen in ein Nasenloch platziert wurde. Neben nasaler Sekretion, Niesen und Juckreiz ergab die Rhinomanometrie einen vierfach erhöhten nasalen Atemwegswiderstand [23].
In 12 Fällen konnten in den Seren von 22 beruflich gegenüber Papain exponierten Arbeitern (keine weiteren Angaben) Papainspezifische IgE-Antikörper mittels CAP nachgewiesen werden. Von diesen enthielten sechs zusätzlich spezifische IgE-Antikörper gegen Latex. Die Tatsache, dass in CAP-Inhibitionsexperimenten Papain zu einer 20 - 33\%igen Hemmung der IgE-Bindung an Latex führte, ließ die Autoren eine gewisse Kreuzreaktivität zwischen den beiden Allergenen vermuten. Dafür spricht auch, dass das Latex-Einzelallergen Hev b 1 und Papain fünf identische Trimere und zwei identische Tetramere in ihren Aminosäuresequenzen aufweisen [24].

Ein 38-jähriger Mann, der erst als Koch und später im Lebensmittelhandel tätig war, klagte über berufsbedingte Atemwegsbeschwerden sowie über Rhinitis und Konjunktivitis beim Umgang mit Papain. Im Hauttest mit $10 \mathrm{mg}$ Papain/ml kam es zu einer sehr deutlichen Reaktion mit einem Quaddeldurchmesser von $9 \mathrm{~mm}$. Mittels EAST konnten Papain-spezifische IgE-Antikörper in hoher Konzentration (RAST-Klasse 4) nachgewiesen werden. Die Spezifität der Reaktion konnte im EAST-Inhibitionstest nicht bewiesen werden, da die Inhibition mit Papain auch bei der Verwendung von an der Festphase gekoppelten Graspollenallergenen nachweisbar war. Dieser Effekt wird von den Autoren durch die proteolytische Zerstörung der IgE-Antikörper durch Papain erklärt [25].

In einer Studie wurden 10 Arbeiter eines pharmazeutischen Unternehmens untersucht, die eine Sensibilisierung auf eines der von ihnen verwendeten Enzyme (Papain, Trypsin, Chymotrypsin, Bromelain, Amylase, Lipase) aufwiesen und von denen fünf über berufsbedingte Beschwerden klagten. Bei acht der 10 sensibilisierten Arbeiter verlief sowohl der Pricktest mit 0,01-1 mg Papain/ml als auch der Nachweis spezifischer IgE-Antikörper mittels RAST positiv. Von den beiden übrigen Beschäftigten reagierte einer ausschließlich im Hauttest positiv während bei dem zweiten Beschäftigten bei negativem Hauttest Papain-spezifische IgE-Antikörper nachgewiesen wurden. Sämtliche Tests verliefen bei 10 nicht-exponierten Kontrollpersonen negativ. Insgesamt konnte eine Sensibilisierung gegen proteolytische Enzyme häufiger beobachtet werden als gegen die Enzyme Amylase und Lipase [25].

Zwei Kosmetikerinnen berichteten über Atemwegsbeschwerden, Rhinitis, Konjunktivitis sowie Kontakturtikaria nach dem Umgang mit papainhaltigen Reinigungstabletten. Der Pricktest mit $5 \%$ iger Papainlösung ergab in beiden Fällen ein positives Ergebnis, ebenso wie der Hauttest (prick to prick) mit Papaya. Papainspezifische IgE-Antikörper waren im Blut beider Patientinnen mittels CAP nachweisbar (47,3 bzw. 19,5 kU/1) [6].

Die Seren von 10 japanischen Köchen, von denen sieben über Beschwerden beim Umgang mit Weizenmehl klagten und neun Weizenmehl-spezifische IgE-Antikörper aufwiesen, wurden mittels RAST auf das Vorhandensein Papain-spezifischer IgE-Antikörper untersucht. In vier Fällen waren spezifische IgE-Antikörper gegen Papain, das für einige Zwecke Mehlen zugesetzt wird, nachweisbar [27]. 


\section{Schlussfolgerung}

Durch eine große Zahl von Studien aus verschiedenen Ländern sind Fälle von spezifischer Überempfindlichkeit der Atemwege oder der Lunge durch die aus der Papaya (Carica papaya) stammende Protease Papain gut belegt. Da Symptome, Hauttestergebnisse, der Nachweis spezifischer IgE-Antikörper und die Ergebnisse spezifischer Provokationstests in der Regel gut übereinstimmen, scheint ein immunologischer Wirkmechanismus gesichert. Außerdem sind auch urtikarielle Sofortreaktionen der Haut durch Kontakt mit Papain möglich. Eindeutige Hinweise auf eine zellvermittelte kontaktallergene Wirkung liegen jedoch nicht vor.

\section{Literatur}

${ }^{1}$ Hoffman A, Henderson AC, Tsai L, Ingenito E. Physiologic responses of sheep to two different methods of papain exposure. Inhal Toxicol 2003; 15: $761-780$

${ }^{2}$ Kampen V van, Merget R. Berufliche Atemwegssensibilisierungen durch Subtilisine. Pneumologie 2002; 56: 182-186

${ }^{3}$ Kampen V van, Lessmann H, Brüning T et al. Berufliche Allergien gegen Cellulasen. Pneumologie 2003; 57: 388 - 391

${ }^{4}$ Kampen V van, Merget R, Brüning T. Berufliche Allergien gegen Xylanasen. Pneumologie 2004; 58: 103 - 106

${ }^{5}$ Baur X, Sander I, Kampen V van. Aerogene Enzyme sind aggressive berufliche Inhalationsallergene. Dtsch Med Wochenschr 2000; 125: 912-917

${ }^{6}$ Soto-Mera MT, Lopez-Rico MR, Filgueira JF et al. Occupational allergy to papain. Allergy 2000; 55: 983-984

${ }^{7}$ Freye HB. Papain anaphylaxis: a case report. Allergy Proc 1988; 9(5): $571-574$

${ }^{8}$ König G, Baur X, Bencze K et al. Papain-Asthma: Untersuchungen zu Häufigkeit und Pathogenese. Prax Klin Pneumol 1981; 35(1): 29-33

${ }^{9}$ Beecher W. Hyperesthetic rhinitis and asthma due to digestive ferments. Illinois Medical Journal 1931; 59: 343 - 344

${ }^{10}$ Milne J, Brand S. Occupational asthma after inhalation of dust of the proteolytic enzyme, papain. Br J Ind Med 1975; 32(4): $302-307$

11 Flindt ML. Respiratory hazards from papain. Lancet 1978; 1(8061): $430-243$

12 Tarlo SM, Shaikh W, Bell B et al. Papain-induced allergic reactions. Clin Allergy 1978; 8(3): 207-215

${ }^{13}$ Novey HS, Marchioli LE, Sokol WN et al. Papain-induced asthma physiological and immunological features. J Allergy Clin Immunol 1979; 63(2): $98-103$
${ }^{14}$ Baur X, Fruhmann G. Papain-induced asthma: diagnosis by skin test, RAST and bronchial provocation test. Clin Allergy 1979a; 9(1): 75-81

${ }^{15}$ Baur X, Fruhmann G. Allergic reactions, including asthma, to the pineapple protease bromelain following occupational exposure. Clin Allergy 1979b; 9(5): $443-450$

${ }^{16}$ Novey HS, Keenan WJ, Fairshter RD et al. Pulmonary disease in workers exposed to papain: clinico-physiological and immunological studies. Clin Allergy 1980; 10(6): 721 - 731

17 Baur X, König G, Bencze K et al. Clinical symptoms and results of skin test, RAST and bronchial provocation test in thirty-three papain workers: evidence for strong immunogenic potency and clinically relevant 'proteolytic effects of airborne papain'. Clin Allergy 1982; 12(1): 9-17

${ }^{18}$ Vogelmeier C, Baur X, König G et al. Spezifische IgG- und IgE-Antikörper bei allergischen Reaktionen der Atemwege auf Papain. Prax Klin Pneumol 1985; 39: $713-714$

${ }^{19}$ Grammer LC, Patterson R. Proteins: chymopapain and insulin. J Allergy Clin Immunol 1984; 74: 635-640

${ }^{20}$ Baur X, Sauer W, Weiss W. Baking additives as new allergens in baker's asthma. Respiration 1988; 54(1): 70-72

${ }^{21}$ Bolm-Audorff U, Bienfait H-G, Hiltl G et al. Atemwegsallergien bei Enzym-exponierten Chemiearbeitern. In: Schuckmann F (Hrsg): Verhandlungen der Deutschen Gesellschaft für Arbeitsmedizin. Stuttgart: AW Gentner, 1991: 201 - 205

${ }^{22}$ Merget R, Stollfuss J, Wiewrodt R et al. Diagnostic tests in enzyme allergy. J Allergy Clin Immunol 1993; 92: 264-277

${ }^{23}$ Niinimaki A, Reijula K, Pirila T et al. Papain-induced allergic rhinoconjunctivitis in a cosmetologist. J Allergy Clin Immunol 1993; 92(3): $492-493$

${ }^{24}$ Baur X, Chen Z, Rozynek P et al. Cross-reacting IgE antibodies recognizing latex allergens, including Hev b 1 , as well as papain. Allergy 1995; 50(7): $604-609$

${ }^{25}$ Merget R, Bergmann E-M, Schärling B. Schwergradiges Berufsasthma durch Papain. Allergo J 1995; 4: 365-369

${ }^{26}$ Zentner A, Jeep S, Wahl R et al. Multiple IgE-mediated sensitizations to enzymes after occupational exposure: evaluation by skin prick test RAST, and immunoblot. Allergy 1997; 52(9): 928-934

${ }^{27}$ Matsumura Y, Niitsuma T, Ito H. A study of factors contributing to bakers' allergy symptoms. Arerugi 1994; 43(5): 625-633

\section{Bereits publizierte Beiträge zu dieser Serie:}

${ }^{1}$ Kühlschmierstoffe. Pneumologie 2003; 57: 212-221

${ }^{2}$ Getreidestaub. Pneumologie 2003; 57: 335-339

${ }^{3}$ Cellulasen. Pneumologie 2003; 57: 388-391

${ }^{4}$ Isocyanate. Pneumologie 2003; 57: 526-531

5 Platinsalze. Pneumologie 2003; 57: 606-611

${ }^{6}$ Nickel. Pneumologie 2003; 57: 667-670

${ }^{7}$ Xylanasen. Pneumologie 2004; 58: $103-106$ 